\title{
Estrategias para el desarrollo cognitivo de un niño en Educación Inicial
}

\author{
Bra. Claudia María Zepeda Padilla, Bra. Elizabeth del Socorro Martínez Andino.
}

Facultad de Educación e Idiomas. ${ }^{1}$

Recibido: 24 abril 2013-Aprobado 28 de octubre 2013.

\section{INTRODUCCIÓN}

En las escuelas, es recurrente ver la falta de atención del docente ante una situación que de alguna manera es un denominador común en todas las escuelas. Nos referimos a los problemas de comportamiento, falta de interés de los niños para realizar determinada actividad, la necesidad de desarrollar las habilidades y destrezas pertinentes para lograr un desarrollo adecuado. Esto conlleva a que el niño sienta que no puede alcanzar un desempeño satisfactorio lo cual afecta su autoestima y por tanto se frustra al adquirir un pensamiento pobre, desmotivador y estancado por las circunstancias del entorno.

El desarrollo cognitivo forma parte esencial dentro del desarrollo del currículo de Educación Inicial. Esto demanda que los docentes durante el proceso de enseñanza-aprendizaje empleen estrategias y recursos didácticos adecuados destinados a potenciar el desarrollo del pensamiento lógico. Por lo que es necesario planificar las actividades con secuencia lógica que permita alcanzar las competencias pertinentes de acuerdo a su nivel para tener éxito en todo su desarrollo escolar. Es necesario proponer a los niños y niñas situaciones didácticas contextualizadas donde se tomen en cuenta sus experiencias, conocimientos previos y plantearle nuevas situaciones en las que ellos puedan comparar, establecer relaciones, transformar, analizar, anticipar resultados, conocer el procedimiento a seguir para resolver problemas, razonar y justificar resultados.

Para detectar el caso de esta investigación, se realizó primero, tres observaciones no participantes durante el desarrollo de la jornada escolar dentro del salón de clase. De acuerdo a las actividades que desarrollaba la maestra con los estudiantes, se notó que algunos niños tenían dificultades para prestar atención y realizar las actividades orientadas por la docente. Estos solicitaban ayuda y no eran atendidos y al final hacían las actividades como les parecía o no la hacían. Luego, se realizó una entrevista a la docente para que opinara en relación a qué niños consideraba con dificultades y por qué. Algunas respuestas brindadas por la docente surgió el nombre del niño dentro del grupo detectado. Con las observaciones realizadas y la entrevista a la maestra se decidió tomar el caso específico de un niño de 5 años de III etapa para quien se realizó un diagnóstico primeramente y de acuerdo a los resultados del mismo, diseñar un plan de acción que responda a sus necesidades de aprendizaje, ejecutarlo y evaluarlo.

\section{DESARROLLO}

\section{Puesta en marcha de la acción}

El método para ejecutar el plan de acción ha sido ejercicios prácticos, como el tipo de enfoque de la investigación lo indica "ACCIÓN", con un procedimiento de observación, conversación, demostración y vivencia. El plan de acción se realizó en cuatro sesiones donde se estableció una comunicación con la docente previamente para la ejecución de las actividades planificadas.

\footnotetext{
${ }^{1}$ Trabajo dirigido por la maestra Valinda del Rosario Sequeira Calero
} 


\begin{tabular}{|c|c|c|}
\hline OBJETIVOS & ACTIVIDADES & EVIDENCIAS/ SEGUIMIENTO \\
\hline $\begin{array}{l}\text { Conoce y practica los } \\
\text { procesos básicos del } \\
\text { desarrollo cognitivo al } \\
\text { colorear y trazar } \\
\text { diferentes líneas con } \\
\text { una intervención } \\
\text { personalizada. }\end{array}$ & $\begin{array}{l}\text { * Apoyo directo al niño dentro del grupo } \\
\text { para realizar sus actividades orientadas por } \\
\text { la docente. } \\
* \text { La docente Claudia orienta colorear las } \\
\text { verduras. } \\
* \text { Luego, pasan a trazar líneas horizontales, } \\
\text { verticales en el libro de caligrafía con la } \\
\text { figura de una casita. }\end{array}$ & $\begin{array}{l}* \text { Toma el crayón correctamente. } \\
* \text { Coloca el espacio gráfico (cuaderno) } \\
\text { con la inclinación adecuada. } \\
* \text { Colorea haciendo movimiento } \\
\text { circular y no rayado. } \\
* \text { Trabaja con orden y limpieza. }\end{array}$ \\
\hline \multirow[t]{2}{*}{$\begin{array}{l}\text { Reconoce las figuras } \\
\text { geométricas básicas y } \\
\text { las líneas curvas. }\end{array}$} & $\begin{array}{l}\text { * Saludo a los niños con alegría y les } \\
\text { explico lo que se hará. } \\
\text { * Presento a los niños las figuras } \\
\text { geométricas una a una yido las mencionen. } \\
* \text { Establezco un diálogo con los niños y } \\
\text { reconociendo características de cada una de } \\
\text { las figuras geométricas presentadas. } \\
\text { * Entono el canto "las figuras" y "los } \\
\text { deditos" para identificar cada una de los } \\
\text { nombres de los dedos y los que se utilizan al } \\
\text { tomar el crayón. } \\
\text { * Explico a los niños lo que realizarán y la } \\
\text { forma cómo se debe hacer. } \\
\text { * Coloreo cada una de las figuras. } \\
\text { * Trazo el borde de cada figura siguiendo la } \\
\text { dirección indicada. } \\
\text { * Luego, salimos al patio y entonamos el } \\
\text { canto las figuras y cada uno menciona la } \\
\text { figura que prefiere y la señala. }\end{array}$ & $\begin{array}{l}\text { * Identifica las formas básicas: círculo, } \\
\text { triángulo, cuadrado, rectángulo. } \\
* \text { Colorea de forma circular, sin salirse } \\
\text { del espacio. } \\
\text { * Traza el borde de la figura siguiendo } \\
\text { la dirección. } \\
\text { * Participa alegremente en la actividad. }\end{array}$ \\
\hline & $\begin{array}{l}\text { * Explico que las líneas que están dibujadas } \\
\text { en el patio se llaman curvas y cómo } \\
\text { realizaremos la actividad. } \\
\text { * En fila de niñas y niños camino sobre las } \\
\text { líneas curvas que están trazadas con cenizas } \\
\text { en el suelo del patio. } \\
\text { * En bandejitas de arena con el dedo índice } \\
\text { trazo las líneas curvas por donde caminé. } \\
\text { * En mi hoja de aplicación, con crayón } \\
\text { trazo las líneas curvas, después las vuelvo a } \\
\text { trazar con lápiz. }\end{array}$ & $\begin{array}{l}* \text { Participa activamente de la actividad } \\
\text { al caminar sobre la línea dibujada en el } \\
\text { patio. } \\
* \text { Usa el dedo índice y traza la línea } \\
\text { curva siguiendo la dirección del trazo. } \\
* \text { Usa correctamente el lápiz y traza la } \\
\text { curva siguiendo la dirección correcta sin } \\
\text { levantar el lápiz. }\end{array}$ \\
\hline $\begin{array}{l}\text { Emplea correctamente } \\
\text { los instrumentos } \\
\text { gráficos del crayón, } \\
\text { lápiz y papel al colorear } \\
\text { figuras geométricas } \\
\text { básicas y controla la } \\
\text { dirección de trazado de } \\
\text { líneas curvas. }\end{array}$ & $\begin{array}{l}* \text { De forma personalizada trabajo con las } \\
\text { figuras geométricas identificándolas al } \\
\text { colorear y trazando el borde siguiendo la } \\
\text { dirección. } \\
\text { * Trazo líneas curvas siguiendo la dirección } \\
\text { correcta. }\end{array}$ & $\begin{array}{l}\text { * Colorea las figuras sin salirse del } \\
\text { espacio. } \\
* \text { Reconoce las figuras geométricas. } \\
* \text { Usa correctamente el espacio y el } \\
\text { instrumento gráfico. } \\
* \text { Traza las líneas curvas siguiendo la } \\
\text { dirección correcta y sin levantar el lápiz. }\end{array}$ \\
\hline $\begin{array}{l}\text { Discrimina figura- } \\
\text { fondo- color y el } \\
\text { lenguaje a través de } \\
\text { preguntas de } \\
\text { comprensión. }\end{array}$ & $\begin{array}{l}\text { Saludo con alegría a Joaquín. } \\
\text { En una mesa dispongo mecate rojo, azul y } \\
\text { verde. } \\
\text { Explico las actividades a realizar: } \\
\text { Tomar el mecate rojo y en el suelo } \\
\text { formar un círculo, luego con el mecate } \\
\text { azul formar un cuadrado y con el mecate } \\
\text { verde formar un triángulo. Las tres }\end{array}$ & $\begin{array}{l}\text { * Identifica los colores rojo, azul y } \\
\text { verde. } \\
* \text { Emplea correctamente los } \\
\text { movimientos de sus dedos y } \\
\text { coordinación óculo-manual para formar } \\
\text { las figuras. } \\
* \text { Mantiene el equilibrio al caminar } \\
\text { sobre las cuerdas según los colores y }\end{array}$ \\
\hline
\end{tabular}




\begin{tabular}{|l|l|l|}
\hline & $\begin{array}{l}\text { figuras deben ir sobrepuestas. } \\
\text { Después de formadas caminar sobre cada } \\
\text { una de ellas según se le vaya indicando. }\end{array}$ & $\begin{array}{l}\text { formas. } \\
* \text { Menciona qué otros objetos son de } \\
\text { color azul, rojo y verde. } \\
* \text { Colorea correctamente en la hoja de } \\
\text { aplicación discriminando las figuras. }\end{array}$ \\
\hline
\end{tabular}

\section{Recogida de datos}

Para realizar todo este proceso los aspectos que favorecieron el trabajo de campo realizado fueron la acogida por parte del centro y la disposición de los informantes claves, como también la disponibilidad de recursos para la realización de las entrevistas y aplicación de las actividades planificadas. Para la recogida de datos se utilizó Diario de Campo y a través de las evidencias/seguimiento del plan de acción que son los criterios para evaluar el alcance de los objetivos planificados, se recopiló lo siguiente:

\begin{tabular}{|c|c|c|}
\hline DÍA \#1 & DÍA \#2 & DÍA \#3 \\
\hline $\begin{array}{l}\text { * Oriento cómo tomar el crayón, la } \\
\text { ubicación del cuaderno y cómo debe } \\
\text { colorear la figura. } \\
\text { El niño colorea rayando, se sale } \\
\text { mucho del borde, lo que quiere decir } \\
\text { que no controla el espacio. } \\
\text { En esta etapa él ya debe tener } \\
\text { control del espacio y colorear de } \\
\text { forma circular hacia adentro. } \\
\text { De una manera amistosa le ayudo al } \\
\text { niño a ubicar su libro y le explico } \\
\text { porque se debe colocar de esa } \\
\text { manera. Luego la forma de tomar el } \\
\text { lápiz y le muestro la dirección al } \\
\text { trazar las líneas de la casita. } \\
\text { Comienza a hacerlas correctamente, } \\
\text { pero después como que se } \\
\text { desconcentra y vuelve a hacer } \\
\text { dispersamente sin seguir la } \\
\text { dirección adecuada de los trazos. } \\
\text { No tiene orden ni limpieza al } \\
\text { trabajar. }\end{array}$ & $\begin{array}{l}\text { * En el momento del diálogo } \\
\text { participó, pero solamente } \\
\text { reconoció el círculo, las otras } \\
\text { figuras, decía los nombres pero no } \\
\text { las identificaba. } \\
\text { El niño coloreo, pero en todas las } \\
\text { figuras se salió del borde, no siguió } \\
\text { la dirección del trazado de los } \\
\text { bordes de la figura. } \\
\text { Para concluir la actividad al } \\
\text { mostrar su trabajo entonando el } \\
\text { canto, no participó. Le pedí que } \\
\text { pasara pero no expresó nada. } \\
\text { Al pasar a trabajar con la arena en } \\
\text { las bandejitas, le costaba usar el } \\
\text { dedo índice y trazar según la } \\
\text { dirección la línea curva. Le mostré } \\
\text { como hacerlo y lo repitió varias } \\
\text { veces hasta lograrlo. } \\
\text { Al trabajar en la hoja de aplicación } \\
\text { observé que comprendió como } \\
\text { trazar la línea curva, aunque le } \\
\text { cuesta un poco hacer la curva, pero } \\
\text { si comprendió el proceso del uso } \\
\text { del crayón y el lápiz. }\end{array}$ & $\begin{array}{l}\text { * Este día trabajé directamente con } \\
\text { Joaquín, recordamos las figuras } \\
\text { geométricas y le pedí nombrara cada } \\
\text { una de ellas y lo hizo. } \\
\text { Nuevamente le expliqué la forma de } \\
\text { tomar el crayón y el movimiento } \\
\text { para colorear. Lo hizo, coloreo } \\
\text { mejor que la vez anterior, de hecho } \\
\text { lo hizo bastante bien, ya que en } \\
\text { algunos momentos hacía el } \\
\text { movimiento al contrario, como que } \\
\text { se le olvidaba. } \\
\text { Las líneas curvas en la hoja de } \\
\text { aplicación lo hizo muy bien. } \\
\text { Siguiendo el movimiento y postura } \\
\text { adecuada. }\end{array}$ \\
\hline
\end{tabular}

\begin{tabular}{|l|l|}
\hline \multicolumn{1}{|c|}{ DIA \#4 } & \multicolumn{1}{c|}{ DIA \#5 } \\
\hline Este día trabajamos de forma personalizada con Joaquín. & Siempre trabajamos de forma personalizada. \\
Identifica los colores rápidamente, se muestra seguro. & $\begin{array}{l}\text { Con alegría va formando los conjuntos clasificándolos } \\
\text { en las cajitas de acuerdo a las formas. }\end{array}$ \\
$\begin{array}{l}\text { Al comenzar a hacer las figuras con el mecate se le } \\
\text { dificulta, no tiene idea de cómo empezar. Muestra } \\
\text { dificultad en los dedos para ir acomodando la cuerda y }\end{array}$ & $\begin{array}{l}\text { Con ayuda nuestra, a través de las preguntas, establece } \\
\text { las semejanzas y las diferencias. }\end{array}$ \\
\hline
\end{tabular}


formar la figura, pero después poco a poco lo logra hacer con mayor habilidad hasta formarla todas.

Responde tímidamente a las preguntas realizadas. Colorea discriminando las figuras correctamente y sin salirse del espacio.
Compara los conjuntos identificando donde hay más, menos, igual. Al mismo tiempo coloca el signo matemático correspondiente. Cabe señalar que no conocía estos signos.

\section{Reflexión y Evaluación}

La evaluación que a continuación se presenta se llevó a cabo partiendo del objetivo general y de los objetivos específicos planteados en este estudio de caso:

Toda niña o niño puede crecer y desarrollarse mejor si se le ayuda, si se le estimula. El objetivo fundamental es el de fortalecer todas aquellas debilidades que ellos no han logrado desarrollar en el seno familiar. Además es importante desde las aulas educativas contribuir al desarrollo afectivo para que el niño y la niña crezcan sanos y felices. (Amor para los más chiquit@s, 2010: 60).

Los padres son los primeros educadores de sus hijos. La función central de familia es la protección y socialización de los que la integran, el docente de educación inicial, por la edad de los niños que tiene a su cargo, es quien más se relaciona con los padres. (Ramírez, 1998: 102.).

La planificación correcta de las actividades en los juegos infantiles requiere el dominio pleno del educador. Además, debe saber cuáles son las actividades que el niño prefiere, cuál es su contenido, quien quiere jugar con quien, hasta qué punto los niños son independientes en los juegos, le es necesario conocer cómo se desarrollan los niños en los juegos infantiles para una edad u otra como cambia y se enriquece el contenido, cuál debe ser la conducta de los niños en el juego, como desarrollar su independencia. Todo esto es importante porque se planifica, no la actividad lúdica de los niños, si no la actividad pedagógica de la educadora con el criterio de "jugando aprendo". (Romero, 1990: 373)

Joaquín es un niño muy inteligente sólo requiere atención y seguimiento para que pueda desarrollar adecuadamente su aprendizaje. Los niños necesitan afecto, atención, paciencia. Es realmente difícil trabajar con más de 40 niños y niñas y se podría decir que imposible ofrecer la atención adecuada a cada uno. Hay niños y niñas que necesitan más ayuda que otros porque tiene la ayuda necesaria también del hogar, por tanto, es importante considerar y tratar de crear una relación más de comunicación y compromiso con los padres de familia.

Las actividades planificadas realmente contribuyeron para que el niño Joaquín desarrollara su proceso cognitivo de discriminación, atención, memoria e imitación. En el diálogo establecido con él, logró discriminar de acuerdo a colores y formas; desarrolló un poco su atención, puesto que al orientarle comprendía lo que se le decía, sin embargo el tiempo implementado para la atención le cuesta bastante, sus lapsos de atención son bastante cortos y esto lleva también a la concentración, que se le dificulta un poco.

Se le estimuló al desarrollo de la memoria asociando colores y formas en relación a las figuras geométricas y su entorno ambiental, por ejemplo: para reconocer el color verde asociarlo con el color de las hojas de los árboles, el color amarillo al sol, el cielo color azul, las formas de los árboles, nubes, sol, etc. Luego solicitarle colorear de un color específico determinada figura o un objeto de acuerdo a la realidad, escogiendo el crayón del color indicado. También se trabajó la imitación porque de acuerdo a los modelos planteados en las hojas de aplicación reprodujo el trazo de las formas geométricas y las líneas curvas siguiendo la dirección correcta y la forma de colorear para tener un producto mejor. En las hojas 
de aplicación se puede notar la diferencia del primer trabajo que se hizo en general como el segundo trabajo de las mismas actividades de forma individualizada.

Desde el punto de vista de Zianny M. (1994) con el desarrollo cognitivo logramos la transformación del desarrollo de habilidades y destrezas en el niño y la niña por medio de la adquisición de experiencias y aprendizajes para su adaptación al medio, implicando procesos de discriminación, atención, memoria, imitación, conceptualización y resolución de problemas, tomando en cuenta que uno de los desempeños que debe haber alcanzado a la edad de los cinco años.

\section{CONCLUSIONES}

Las condiciones familiares en las que se desarrolla Joaquín son bastante difíciles, en el día su mamá y papá se van a trabajar. Prácticamente queda al cuido de su abuelita, por tanto en el día no tiene ninguna disciplina de estudio, ni organización de actividades. La atención que brinda la docente no es adecuada, ya que tiene a su cargo 42 niños, sin embargo, consideramos no es motivo para no ofrecer el cuidado adecuado y pertinente para el proceso enseñanza-aprendizaje con los párvulos.

El plan elaborado y ejecutado dio respuesta a ciertas necesidades, tratando de desarrollar habilidades básicas que le sean de utilidad en su proceso enseñanza aprendizaje. De hecho, aunque Joaquín fue atendido en el área de desarrollo cognitivo, no quiere decir que no necesitara ayuda en otras áreas, especialmente en la socialización y la afectividad. Sin embargo, con la relación establecida con el niño, indirectamente se trabajaron estas áreas. El niño mostró cambio y adquirió cierta habilidad de acuerdo a las estrategias y acciones planteadas, aunque el tiempo fue muy poco, por tanto, se puede constatar y afirmar que los niños y niñas son capaces de aprender si se les da la atención adecuada a través de una intervención pedagógica.

\section{BIBLIOGRAFÍA}

Rodríguez de la Torre, Arturo (Diciembre 1997. Pág. 6). El desarrollo del pensamiento lógicomatemático. Concreción presentada en Congreso.

Rojas Poveda, Luisa Mireya. Tijerino Mora, Anabell de Fátima. Murillo Valdivia, Helia María (2009). Programa de Educación Inicial (3 a 6 años). Managua, Nicaragua.

Rojas Zamora, Marisela (2008). Educación científica y matemática para el niño preescolar I; perspectiva constructivista. Editorial EUNED. San José, Costa Rica.

Spiegel, Alejandro (2006). Recursos didácticos y formación profesional por competencias: Orientaciones metodológicas para su selección y diseño. Buenos Aires.

Tortella, Jame (1981). Las seis etapas del aprendizaje en matemáticas. (4ta ed). Barcelona: Teide

Zianny M. Gamboa (1994). Educación científica y matemática para el niño prescolar. San José Costa Rica: Editorial Universidad estatal a distancia. 